\title{
Long-term Outcomes of Elders Discharged on Antipsychotics
}

\author{
Kah Poh Loh, BMedSci, MBBCh BAO'*, Sheryl Ramdass, BMedSci, MBBCh BAO², Jane L. Garb, MS³, Monica Thim, DO², \\ Maura J. Brennan, MD ${ }^{4,5}$, Peter K. Lindenauer, MD, MSc ${ }^{2,4,6}$, Tara Lagu, MD, MPH ${ }^{2,4,6}$
}

\begin{abstract}
${ }^{1}$ Division of Hematology/Oncology, James P. Wilmot Cancer Institute, University of Rochester/Strong Memorial Hospital, Rochester, New York; ${ }^{2}$ Department of Medicine, Baystate Medical Center, Springfield, Massachusetts; ${ }^{3}$ Division of Academic Affairs, Baystate Medical Center/Tufts University School of Medicine, Springfield, Massachusetts; ' ${ }^{4}$ epartment of Medicine, Tufts University School of Medicine, Springfield, Massachusetts; ${ }^{5}$ Division of Geriatrics, Palliative Care and Post-Acute Medicine, Baystate Medical Center, Springfield, Massachusetts; ${ }^{6}$ Center for Quality of Care Research, Baystate Medical Center, Springfield, Massachusetts.
\end{abstract}

BACKGROUND: Despite limited evidence of efficacy, antipsychotics (APs) are commonly used to treat delirium. There has been little research on the long-term outcomes of patients who are started on APs in the hospital.

METHODS: Using a previously described retrospective cohort of 300 elders ( $\geq 65$ years old) who were newly prescribed APs while hospitalized between October 1, 2012 and September 31, 2013, we examined the 1-year outcomes of patients alive at the time of discharge. We examined number of readmissions, reasons for readmission, duration of AP therapy, use of other sedating medications, and incidence of readmission. We used the National Death Index to describe 1-year mortality and then created a multivariable model to identify predictors of 1-year mortality.

RESULTS: The 260 patients discharged alive from their index admissions had a 1-year mortality rate of $29 \%$ (75/
260). Of the $146 / 260$ patients discharged on APs, 60 (41\%) patients experienced at least 1 readmission. At the time of first readmission, $65 \%$ of patients were still taking the same APs on which they had been discharged. Eighteen patients received new APs during the readmission hospitalizations. Predictors of death at 1 year included discharge to postacute facilities after index admission (odds ratio [OR]: 2.28; 95\% confidence interval $[\mathrm{CI}]:$ 1.10-4.73, $P=0.03$ ) and QT interval prolongation $>500$ ms during index admission (OR: $3.41 ; 95 \% \mathrm{Cl}: 1.34-8.67, P=0.01)$.

CONCLUSIONS: Initiating an AP in the hospital is likely to result in long-term use of these medications. Patients who received an AP during a hospitalization were at high risk of death in the following year. Journal of Hospital Medicine 2016;11:550-555. (c) 2016 Society of Hospital Medicine
Delirium, a clinical syndrome characterized by inattention and acute cognitive dysfunction, is very common in older hospitalized patients, with a reported incidence of $18 \%$ to $35 \%$ at time of admission and overall occurrence rates of $29 \%$ to $64 \% .^{1}$ Previous studies have reported that a diagnosis of delirium is not benign and is associated with other adverse outcomes including prolonged hospitalization, institutionalization, increased cost, and mortality. These outcomes occurred independent of age, prior cognitive functioning, and comorbidities. ${ }^{2}$ Guidelines recommend that management of inpatient delirium should be focused on addressing the underlying etiology and managed with nonpharmacological interventions whenever possible. ${ }^{3-5}$ However, implementing these recommendations can prove to be very challenging in hospital settings. Providers frequently have to resort to medical therapies, including antipsychotics (APs).

*Address for correspondence and reprint requests: Kah Poh Loh, BMedSci, James P. Wilmot Cancer Institute, University of Rochester Medical Center, 601 Elmwood Avenue, Box 704, Rochester, NY 14642; Telephone: 413-306-9767; Fax: +1585-273-1042; E-mail: melissalkp@ gmail.com

Additional Supporting Information may be found in the online version of this article.

Received: September 8, 2015; Revised: February 11, 2016; Accepted: February 20, 2016

2016 Society of Hospital Medicine DOI 10.1002/jhm.2585

Published online in Wiley Online Library (Wileyonlinelibrary.com).
Although these medications are commonly used to treat delirium in elderly patients, there is limited evidence to support their efficacy, and there are currently no proven pharmacological alternatives to these medications. ${ }^{6}$ Furthermore, previous studies have demonstrated an increased risk of stroke, infection, cognitive impairment, and mortality in elders with dementia who receive long-term AP therapy. ${ }^{7-9}$ Yet as many as $48 \%$ of hospitalized elders who were newly started on APs had these drugs continued at time of discharge. ${ }^{10}$

There have been few studies describing the longterm outcomes of elderly patient who are started on APs in the hospital. Most information on outcomes comes from patients with dementia. Therefore, we studied the 1-year outcomes of a cohort of patients with and without dementia who were started on APs in the hospital and then discharged on these medications. In this cohort, we aimed to describe the number of readmissions, reasons for readmissions, duration of AP therapy, use of other sedating medications such as anxiolytics, hypnotics, and antihistamines as well as the incidence of readmission and death 1 year after the index hospital discharge.

\section{METHODS}

We previously described a retrospective cohort of 300 elders ( $\geq 65$ years old) admitted to a tertiary care hospital between October 1, 2012 and September 31, 2013 who were newly prescribed APs while 


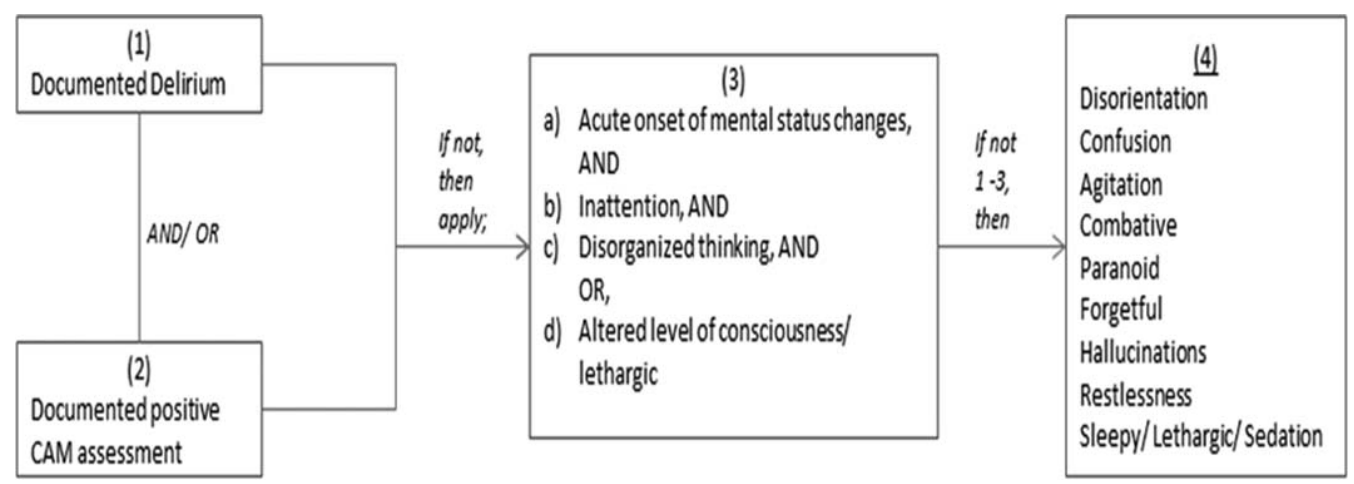

FIG. 1. Methodology for defining delirium during chart review. Abbreviations: CAM, Confusion Assessment Method.

hospitalized. ${ }^{10}$ Of patients alive at the time of discharge (260), 56\% (146 patients) were discharged on APs. Two investigators extracted these 148 patient charts independently to identify and quantify the number of readmissions to the index hospital. We then limited the sample to only the first readmission per patient following the index admission and extracted this readmission for each patient. We first determined if APs were present on the admission medication reconciliation. If APs were not present on admission, we examined whether they were resumed during the hospitalization using the electronic medication administration summary. If they were present on admission, we looked to see if they were discontinued during the readmission and if additional new APs were started during the hospitalizations. We documented the circumstances around APs use and identified patients who died during their hospitalizations. We identified delirium using the same terms that were described in our prior study on the same cohort of patients. ${ }^{10} \mathrm{We}$ determined if patients were delirious using a predetermined algorithm (Figure 1). Briefly, we first determined delirium was documented. We then examined whether there was a Confusion Assessment Method (CAM) instrument included in the record. If a CAM instrument was not documented, we then looked for documentation using specific terms (eg, disorientation, confusions). We identified patients with dementia by determining whether dementia was documented along with other admission medical comorbidities. If it was not, we determined whether dementia was newly diagnosed during the hospital stay using progress notes or consultation notes. We did not objectively define criteria for diagnosis of dementia. We used the National Death Index (NDI) to determine mortality for all patients 1 year after discharge from the index hospitalization. The NDI is a national database of death records maintained by the National Center for Health Statistics. It has shown consistently high sensitivity and specificity for detection of death. ${ }^{11}$

We used descriptive statistics (means, standard deviations, range, and percents as appropriate to the scale of measurement) to describe the patient sample. We then used multiple logistic regression to identify significant predictors of death within 1 year of discharge. ${ }^{12}$ Univariate analysis was used to select candidates for the logistic model ( $t$ tests for continuous factors and $\chi^{2}$ for discrete factors). All factors with a significance level $<0.2$ on univariate analysis were included in the logistic regression, in addition to age and sex (regardless of significance). A maximum likelihood procedure was used to calculate the regression coefficients for the logistic model. The likelihood ratio criterion was used to determine the significance of individual factors in the regression model. ${ }^{13}$ Factors with a significance level of 0.15 or less were retained in the final model, in addition to age and sex.

\section{RESULTS}

The 260 patients discharged alive from their index admissions had a 1-year mortality rate of $29 \%$ (75/ 260). Of the $146 / 260$ patients discharged on APs, 60 $(41 \%)$ patients experienced at least 1 readmission (mean $=2$ readmissions per patient; range, 1-8, with 111 total readmissions for 60 patients) within 1 year from discharge (Figure 2). Most common diagnoses at the time of readmissions were related neurological and psychiatric disorders $(14 \%)$, cardiovascular and circulation disorders $(13 \%)$, renal injury and electrolyte disorders $(11 \%)$, and infections (6\%). Among patients with at least 1 readmission, the mean age was 81.3 (range, 65.5-99.7), 60\% were male, and $45 \%$ were admitted from a skilled nursing facility or rehabilitation facility (Table 1). Median time to readmission was 43.5 days (range, 1-343 days), and 79\% were readmitted to a medical service. The remaining $20 \%$ were admitted to a surgical service. Inpatient mortality during first readmissions was $8 \%$ (5/60). At the time of first readmission, $39 / 60(65 \%)$ of patients were still on the same APs on which they had been discharged, and the APs were continued during the hospitalization in $79 \%$ of the patients $(61 \%$ quetiapine, $19 \%$ olanzapine, and $13 \%$ risperidone). About half of patients whose APs were discontinued prior to readmission received a new AP during their hospital 


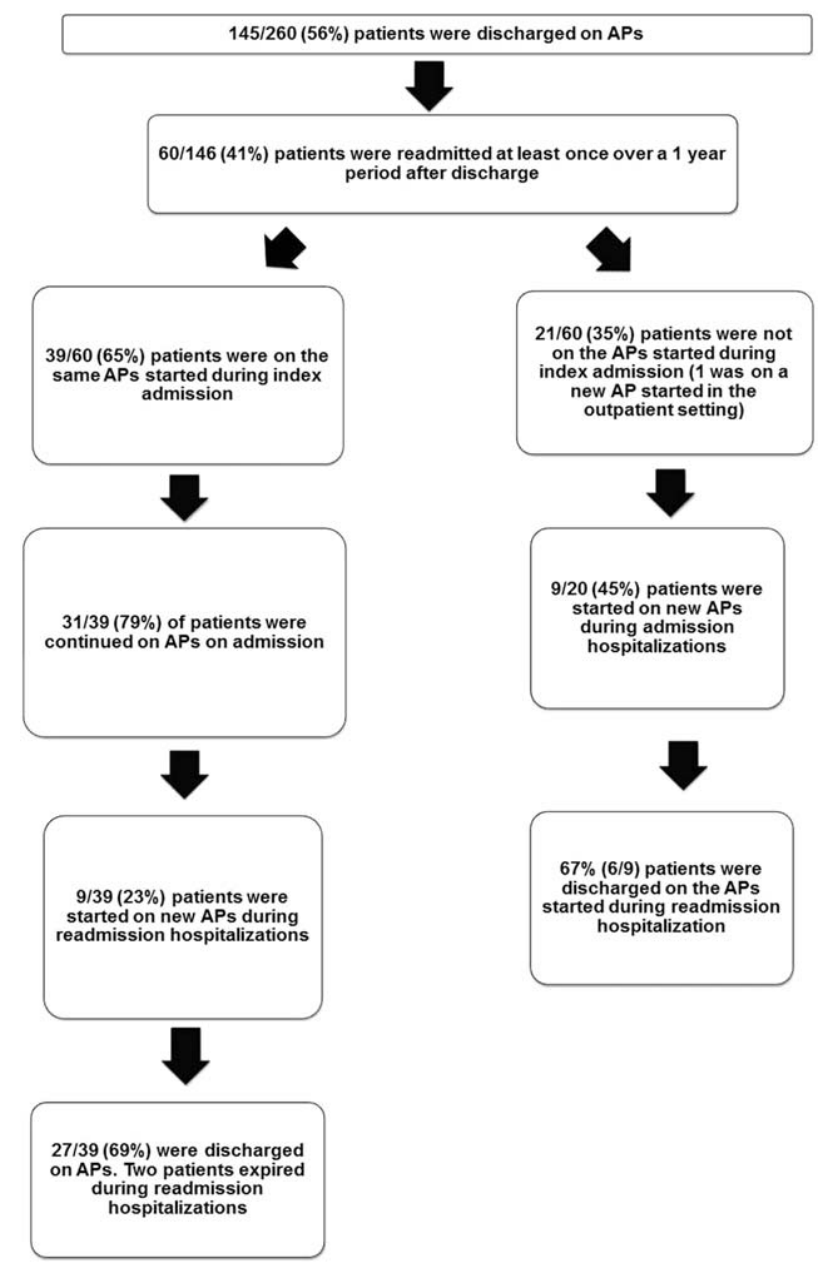

FIG. 2. Flowchart showing the outcomes of patients who were discharged on antipsychotics during index hospitalization and readmitted within 1 year from discharge. Abbreviations: APs, antipsychotics.

stays $(9 / 20 ; 45 \%)$. One patient had been started on quetiapine in the outpatient setting. No patients were found to have new benzodiazepines, nonbenzodiazepine hypnotic, or antihistamines on their admission medication list.

Eighteen patients received 1 or more new APs during the readmission hospitalizations. These included haloperidol (89\%) and quetiapine (39\%). Delirium was the main reported indication for starting APs $(78 \%)$, but in $17 \%$ of cases no indication was documented. An electrocardiogram (ECG) was performed in $94 \%$ prior to APs administration and for $22 \%$ after APs administration. Corrected QT interval (QTc) of $>500 \mathrm{~ms}$ was present in $18 \%$ of patients in pretreatment ECG and $50 \%$ of patients in post-AP ECG. Of patients who survived readmission, 58\% (32/55) were discharged to postacute facilities. Of the 39 patients who were on the same APs from index admission, 27 $(69 \%)$ patients were eventually discharged on the same APs or new APs started during the readmission.

In the multivariable model (Table 2), predictors of death at 1 year included discharge to postacute facilities after index admission (odds ratio [OR]: 2.28; $95 \%$ confidence interval $[\mathrm{CI}]: 1.10-4.73, P=0.03)$
TABLE 1. Demographic Data of the Patients Who Were Discharged on APs and Were Readmitted the First Time Within One Year From Discharge and Circumstances Surrounding APs Initiation During Readmission

\begin{tabular}{|c|c|}
\hline Variables & Value $^{*}$ \\
\hline Age, mean (range), yr & $81.3(65.5-99.7)$ \\
\hline \multicolumn{2}{|l|}{ Gender, no. $(\%)$} \\
\hline Male & $36(60)$ \\
\hline Female & $24(40)$ \\
\hline \multicolumn{2}{|l|}{ Admitted from, no. $(\%)$} \\
\hline Home & $33(55)$ \\
\hline Rehabilitation facilities & $5(8)$ \\
\hline SNF & $22(37)$ \\
\hline \multicolumn{2}{|l|}{ Services, no. $(\%)$} \\
\hline Medicine & $48(80)$ \\
\hline Surgery & $12(20)$ \\
\hline \multicolumn{2}{|c|}{ Types of APs continued on readmission (from index admission), no. (\%)† } \\
\hline Quetiapine & $19(61)$ \\
\hline Olanzapine & $6(19)$ \\
\hline Risperidone & $4(13)$ \\
\hline Haloperidol & $2(7)$ \\
\hline \multicolumn{2}{|c|}{ Types of APs started during readmission, no. (\%)‡ } \\
\hline Quetiapine & $7(39)$ \\
\hline Risperidone & $2(11)$ \\
\hline Haloperidol & $16(89)$ \\
\hline \multicolumn{2}{|l|}{ Indications for AP use, no. (\%)‡ } \\
\hline Delirium & $14(77)$ \\
\hline Undocumented & $3(17)$ \\
\hline Other & $1(6)$ \\
\hline \multicolumn{2}{|l|}{ ECG, no. $(\%) \neq$} \\
\hline Prior to APs administration & $17(94)$ \\
\hline After APs administration & $4(22)$ \\
\hline \multicolumn{2}{|c|}{ QTc prolongation > $500 \mathrm{~ms}$, no. $(\%)$} \\
\hline Prior to APs administration§ & $3(18)$ \\
\hline After APs administration | & $2(50)$ \\
\hline \multicolumn{2}{|l|}{ Discharge destination, no. (\%)ף } \\
\hline Home & $23(38)$ \\
\hline Rehabilitation facilities & $4(7)$ \\
\hline SNF & $28(47)$ \\
\hline Death & $5(8)$ \\
\hline
\end{tabular}

NOTE: Abbreviations: APs, antipsychotics; ECG, electrocardiogram; QTc, QT interval; SNF, skilled nursing facility. ${ }^{*} \mathrm{~N}=60$; patients who were discharged on APs during index admission and were readmitted within 1 year from discharge (only first readmission was included). †Denominator $=31$; number of patients whom APs from index admission were continued during readmission. $\neq$ Denominator $=18$; number of patients who were started on APs during readmission. §Denominator $=17$; number of patients ECG was performed prior to APs administration; "|Denominator = 4; number of patients ECG was performed after APs administration. १Denominator = 55; number of patients who were alive during readmission

and QTc prolongation $>500 \mathrm{~ms}$ during index admission (OR: 3.41; 95\% CI: 1.34-8.67, $P=0.01$ ). Age and gender were not associated with 1-year mortality.

\section{DISCUSSION}

In a cohort of elderly patients who were discharged on APs, nearly one-third (29\%) died within 1 year of the hospitalization in which APs were initiated. Nearly half of the survivors from the index admission $(41 \%)$ experienced at least 1 admission within 1 year from discharge. Of readmitted patients, two-thirds were taking the same APs that had been started during the index hospitalization. Half of the patients not on APs on readmission were started on an AP during the hospitalization, most often because they became 
TABLE 2. Multivariable Analysis of One-Year Mortality From Index Admission in Patients Who Were Started on Antipsychotics.

\begin{tabular}{lccc}
\hline & $\begin{array}{c}\text { Odds } \\
\text { Ratio }\end{array}$ & $\begin{array}{c}95 \% \text { Confidence } \\
\text { Interval }\end{array}$ & P Value \\
\hline Age & 1.03 & $0.99-1.06$ & 0.13 \\
Male sex & 0.87 & $0.50-1.52$ & 0.63 \\
Risperdal & 3.53 & $0.64-19.40$ & 0.15 \\
QTc prolongation after AP administration* & 3.41 & $1.34-8.67$ & 0.01 \\
Presence of geriatric psychiatry consult & 0.30 & $0.09-1.04$ & 0.06 \\
Discharged to postacute facilities vs home & 2.28 & $1.10-4.73$ & 0.03 \\
\hline
\end{tabular}

NOTE: Abbreviations: AP, antipsychotic; QTc, QT interval. *For subset of patients for whom electrocardiogram was done.

delirious on return to the acute care setting. Compared to patients discharged home after an index admission, patients who were discharged to postacute facilities were almost 4 times as likely to die during the year subsequent to the admission. These data suggest that once patients are started on APs, most are continued on them until the next admission or are restarted during that readmission. Moreover, hospitalized elders who require an $\mathrm{AP}$ are at high risk for mortality in the coming year.

Prior studies have reported that patients with delirium have elevated 1-year mortality rates. ${ }^{14-19}$ A secondary analysis of the Delirium Prevention Trial, which included 437 hospitalized older patients, revealed a 1 -year mortality rate of $20 \%$ in those who were never delirious during hospitalization, compared to $26 \%$ to $38 \%$ in patients with delirium. ${ }^{19}$ Additionally, 1-year mortality in hospitalized older patients with delirium (36\%) was shown to be higher than patients with dementia $(29 \%)$ or depression $(26 \%){ }^{17}$ Unlike these studies, not all of the patients in our study had documented delirium, but all received an AP. Still, it is notable that the 1-year mortality rate for delirium in general is similar to what we found in this study.

The literature has also reported that long-term AP use is associated with excess mortality in elder patients, especially those with dementia. ${ }^{20-22}$ In a retrospective cohort study, older patients with dementia who were taking antipsychotics had significantly higher 1-year mortality rates $(23 \%-29 \%)$ than patients not taking antipsychotic medications (15\%). In a large Canadian propensity score-matched cohort study that included over 13,000 demented older adults, the mortality was higher in the communitydwelling elders who received atypical APs compared to no APs, with a difference of $1.1 \%$ in 180 -day mortality rate after initiation of APs. ${ }^{21}$ The absolute mortality rate was $2.6 \%$ higher in patients who received typical compared to atypical APs. Unlike these studies, not every patient in our cohort had a diagnosis of dementia, but again, mortality rates in these studies appear similar to our cohort.
In contrast, other observational studies have not found an increased risk associated with receipt of APs. For example, a prospective study that enrolled approximately 950 patients with probable dementia showed that AP use was not associated with time to death after adjustment for comorbidities, demographic and cognitive variables. ${ }^{23}$ These conflicting results highlight the difficulties of attributing outcomes in high-risk populations. Although the excess mortality observed in patients taking APs may be related to the risks of APs, it is quite possible that patients who require APs (most often for delirium or agitated dementia) are at higher risk of death. This confounding by indication may be nearly impossible to adjust for retrospectively, even using techniques such as propensity matching.

Our report adds to the literature; we know of no studies to date describing a cohort of patients, most with delirium, who were started on APs in the hospital. We also attempted to identify the reasons that patients were started on APs, which have been infrequently reported. As noted above, our 1-year mortality rate of $29 \%$ among older patients prescribed APs in the hospital was quite similar to mortality rates both for patients with delirium who were not necessarily treated with APs and patients with dementia who were treated with APs. This finding further supports the argument that risk factors for mortality, including dementia, delirium, and AP use are very difficult to tease apart. It is possible that the reasons that APs are prescribed (agitated delirium or dementia) have as much to do with the excess mortality reported in observational studies of APs as the use of APs themselves.

The high rate of continued AP use we observed (two-thirds of readmitted patients) may reflect limited pharmacological alternatives to these medications with little evidence to support treating the symptoms of delirium with other drug classes, along with suboptimal environmental and behavioral modifications in postacute facilities and hospitals. This is unfortunate given that delirium is often preventable. Systematic implementation of well-documented strategies to decrease delirium in hospitals and postacute facilities would likely reduce the prescription of APs and has the potential to slow the decline in this vulnerable population. A meta-analysis incorporating both randomized and nonrandomized trials of medical and surgical patients showed that multicomponent nonpharmacologic interventions decreased delirium by $50 \%{ }^{24}$ Thus, simple interventions such as reorientation, early mobilization, optimizing vision and hearing, sleep-wake cycle preservation, and hydration might avoid roughly 1 million cases of delirium in hospitalized older adults annually. ${ }^{24}$ The Hospital Elder Life Program and Acute Care for Elders units are examples of programs that have been shown to decrease the incidence of delirium. ${ }^{25,26}$ 
Despite vigorous efforts to prevent delirium, a subgroup of patients still will become delirious. These patients are at high risk for death. Our mortality prediction model revealed that patients who were discharged to postacute facilities were 4 times more likely to die during the subsequent year compared to patients who were discharged home. Patients discharged to postacute facilities are likely to have a higher burden of disease, greater functional and cognitive impairment, and more frailty than those who are able to return to the community. Very ill and/or frail patients receiving APs in the hospital and requiring APs on discharge to postacute care facilities have limited survival and may benefit from expedited palliative care interventions to clarify prognosis and goals, and relieve suffering. At a minimum, our study identifies a need for further study to identify this very high-risk group of elders. It is notable that $50 \%$ of patients were found to have a post-treatment ECG with a QTc of $>500 \mathrm{~ms}$, a finding that has not been previously described. This would put these patients at higher risk of mortality, and as such we suggest that current guidelines should continue to emphasize the importance of post-treatment ECGs and set clear criteria for discontinuation in elderly patients.

Our study is limited by its retrospective, singlecenter design and small sample size, therefore limiting the interpretation and generalizability of the results to other hospitals. Quetiapine was the most common AP medication used in our hospital; therefore, our findings cannot be generalized to hospitals that utilize other AP agents. Future studies should examine antipsychotic use across hospitals to determine variation in prescribing patterns and outcomes. Nevertheless, the care of these patients were transitioned to a large number of geriatricians and primary care and nursing home physicians after discharge, and the reflected practice patterns extended beyond our hospital. Additionally, we were unable to determine when and why APs were discontinued or started in the outpatient setting. We were only able to detect readmissions to the 3 hospitals within our health system and therefore may have missed some readmissions to other institutions, although the majority of patients in our region tend to return to the same hospital. For patients who were not readmitted, we were also unable to identify whether they remained on the APs initiated during their index hospitalizations. Any retrospective study is limited by the difficulty of distinguishing delirium from the behavioral and psychiatric symptoms of dementia, but we identified delirium using standard terms described in previous literature. ${ }^{10}$ We were unable to determine the types of delirium (hyperactive vs hypoactive) given that the documentations on behavioral symptoms were largely missing from the charts. The number of patients with preexisting diagnosis of dementia was likely underestimated, as we were only able to verify the diagnosis from the medical history. Additionally, the retrospective design based on chart review limited the factors that we could detect and grade accurately for inclusion in our mortality prediction model. Of note, our model did not contain objective measures of cognition, agitation, function, and markers for frailty such as walking speed, weak grip strength, weight loss, and low physical activity.

\section{CONCLUSION}

Initiating an AP (eg, haloperidol, quetiapine, olanzapine, and risperidone) in the hospital is likely to result in long-term use of these medications despite the fact that AP use has been associated with multiple risks including falls, fractures, stroke, cardiovascular disease, and increased mortality in those with underlying dementia. ${ }^{27}$ When possible, behavioral interventions to prevent delirium and slow the trajectory of decline should be implemented to reduce AP use. If patients with delirium are started on antipsychotics, it is important to monitor for prolonged QTc given the associated risk of mortality. In a subgroup of patients at high risk for death in the upcoming year, occurrence of delirium or use of APs during a hospitalization should both be considered triggers for early advance care planning and possibly palliative care and end-of-life discussions, with an emphasis on quality of life.

Disclosures: The research was supported by the Department of Medicine, Baystate Medical Center/Tufts University School of Medicine. Dr. Lagu is supported by the National Heart, Lung, and Blood Institute of the National Institutes of Health under award number K01HL114745. Drs. Lagu and Loh had full access to all of the data in the study. They take responsibility for the integrity of the data and the accuracy of the analysis. Drs. Loh, Brennan, Lindenauer, and Lagu conceived of the study. Drs. Loh, Ramdass, and Ms. Garb acquired the data. Ms Garb analyzed and interpreted the data. Drs. Loh, Ramdass, and Thim drafted the manuscript. Drs. Brennan, Lindenauer, and Lagu and Ms. Garb critically reviewed the manuscript for important intellectual content. Dr. Lagu has received consulting fees from the Institute for Healthcare Improvement, under contract to the Centers for Medicare and Medicaid Services, for her work on a project to help health systems achieve disability competence. Dr. Brennan is supported by a Geriatric Work Force Enhancement Grant from the US Department of Health and Human Services award number 1 U1QHP287020100. The authors report no conflicts of interest.

\section{References}

1. Inouye SK, Westendorp RGJ, Saczynski JS. Delirium in elderly people. Lancet. 2014;383:911-922.

2. Fong TG, Jones RN, Marcantonio ER, et al. Adverse outcomes after hospitalization and delirium in persons with Alzheimer disease. Ann Intern Med. 2012;156:848-856, W296.

3. American Geriatrics Society Expert Panel on Postoperative Delirium in Older Adults. American Geriatrics Society abstracted clinical practice guideline for postoperative delirium in older adults. J Am Geriatr Soc. 2015;63:142-150.

4. Practice guideline for the treatment of patients with delirium. American Psychiatric Association. Am J Psychiatry. 1999;156:1-20.

5. Potter J, George J; Guideline Development Group. The prevention, diagnosis and management of delirium in older people: concise guidelines. Clin Med (Lond). 2006;6:303-308.

6. Seitz DP, Gill SS, van Zyl LT. Antipsychotics in the treatment of delirium: a systematic review. J Clin Psychiatry. 2007;68:11-21.

7. Maust DT, Kim HM, Seyfried LS, et al. Antipsychotics, other psychotropics, and the risk of death in patients with dementia: number needed to harm. JAMA Psychiatry. 2015;72:438-445. 
8. Andrade C, Radhakrishnan R. Safety and efficacy of antipsychotic drugs for the behavioral and psychological symptoms of dementia. Indian J Psychiatry. 2009;51(suppl 1):S87-S92.

9. Gareri P, De Fazio P, Manfredi VGL, De Sarro G. Use and safety of antipsychotics in behavioral disorders in elderly people with dementia. J Clin Psychopharmacol. 2014;34:109-123.

10. Loh KP, Ramdass S, Garb JL, Brennan MJ, Lindenauer PK, Lagu T. From hospital to community: use of antipsychotics in hospitalized elders. J Hosp Med. 2014;9:802-804.

11. Sesso HD, Paffenbarger RS, Lee I-M. Comparison of National Death Index and World Wide Web Death Searches. Am J Epidemiol. 2000; 152:107-111.

12. Cox DR. Analysis of Binary Data. London, United Kingdom: Methuen; 1970:76-99.

13. Lee ET. Statistical Methods for Survival Data Analysis. New York, NY: John Wiley \& Sons; 1992:233-236.

14. Evans SI, Sayers M, Mitnitski A, Rockwood K. The risk of adverse outcomes in hospitalized older patients in relation to a frailty index based on a comprehensive geriatric assessment. Age Ageing. 2014;43: $127-132$.

15. Grover S, Ghormode D, Ghosh A, et al. Risk factors for delirium and inpatient mortality with delirium. J Postgrad Med. 2013;59:263-270.

16. Avelino-Silva TJ, Farfel JM, Curiati JAE, Amaral JRG, Campora F, Jacob-Filho W. Comprehensive geriatric assessment predicts mortality and adverse outcomes in hospitalized older adults. BMC Geriatr. 2014;14:129.

17. Tsai M-C, Weng H-H, Chou S-Y, Tsai C-S, Hung T-H, Su J-A. Oneyear mortality of elderly inpatients with delirium, dementia, or depression seen by a consultation-liaison service. Psychosomatics. 2012;53: 433-438.
18. van Hemert AM, van der Mast RC, Hengeveld MW, Vorstenbosch M. Excess mortality in general hospital patients with delirium: a 5 -year follow-up of 519 patients seen in psychiatric consultation. I Psychosom Res. 1994;38:339-346.

19. McAvay GJ, Van Ness PH, Bogardus ST, et al. Older adults discharged from the hospital with delirium: 1-year outcomes. I Am Geriatr Soc. 2006;54:1245-1250.

20. Ballard C, Hanney ML, Theodoulou M, et al. The dementia antipsychotic withdrawal trial (DART-AD): long-term follow-up of a randomised placebo-controlled trial. Lancet Neurol. 2009;8:151-157.

21. Gill SS, Bronskill SE, Normand S-LT, et al. Antipsychotic drug use and mortality in older adults with dementia. Ann Intern Med. 2007; 146:775-786.

22. Wang PS, Schneeweiss S, Avorn J, et al. Risk of death in elderly users of conventional vs. atypical antipsychotic medications. N Engl J Med. 2005;353:2335-2341.

23. Lopez OL, Becker JT, Chang Y-F, et al. The long-term effects of conventional and atypical antipsychotics in patients with probable Alzheimer's disease. Am J Psychiatry. 2013;170:1051-1058.

24. Hshieh TT, Yue J, Oh E, et al. Effectiveness of multicomponent nonpharmacological delirium interventions: a meta-analysis. JAMA Intern Med. 2015;175:512-520.

25. Rubin FH, Neal K, Fenlon K, Hassan S, Inouye SK. Sustainability and scalability of the hospital elder life program at a community hospital. J Am Geriatr Soc. 2011;59:359-365.

26. Fox MT, Persaud M, Maimets I, et al. Effectiveness of acute geriatric unit care using acute care for elders components: a systematic review and meta-analysis. I Am Geriatr Soc. 2012;60:2237-2245.

27. Muench J, Hamer AM. Adverse effects of antipsychotic medications. Am Fam Physician. 2010;81:617-622. 